\title{
Extinction Learning as a Model of Drug Treatment and Relapse: A Behavioral Overview
}

\author{
John J. Widholm* \\ Department of Psychology, College of Charleston, Charleston, SC 29424, USA
}

\begin{abstract}
Extinction is the process by which a previously established stimulus relationship is broken by the removal of reinforcers and/or biologically relevant stimuli, causing a reduction in responding. Given the importance of this phenomenon in terms of understanding not only learning and behavior, but also of enhancing our understanding of drug addiction and treatment, there is renewed attention being given to the study of extinction in the behavioral, neuroscientific, and therapeutic disciplines. The purpose of the current review is to provide an overview of the basic Pavlovian extinction paradigm and its relevance for treating drug addiction and discuss the typical "threats to extinction" as they model the tendency for drug relapse.
\end{abstract}

Keywords: Pavlovian learning, drug addiction, cue-based therapy, contextual control, spontaneous recovery, renewal, reinstatement, rapid reacquisition.

\section{INTRODUCTION}

Extinction is an important process of learning that is typically defined as the removal of reinforcers and/or biologically relevant stimuli from a previously established stimulus relationship that results in the reduction in responding. This historically important behavioral endpoint [1] is receiving renewed attention [2-7], causing a change in our understanding of the phenomenon. Given the success of applying Pavlovian learning paradigms to our understanding of drug addiction $[8,9]$ and the use of extinction procedures as a therapeutic tool to reduce the motivational salience of drug-related stimuli $[10,11]$ there is an increased interest in studying the neurobiological, neuropharmacological, and neurophysiological correlates of extinction with the goal of improving and/or expediting the extinction process [12-14]. The purpose of the current review is to provide a brief summary of the basic Pavlovian extinction paradigm and its relevance for treating drug addiction and discuss the typical "threats to extinction" as these model (and make predictions about) the tendency for drug relapse.

\section{EXTINCTION (AND ACQUISITION) PARADIGM}

The importance of Pavlov's contribution to the study of psychology cannot be overstated because it provided a formal methodology to understand associative learning processes [1]. Briefly, stimuli that innately elicit a reflexive response are referred to as Unconditional Stimuli (US; they elicit a response without prior conditions or prior learning) and the responses that are elicited are called Unconditional Responses (UR). Some traditional examples of USs (and corresponding URs) include food (salivation), cold (piloerection), and light (pupillary constriction). When neutral stimuli (e.g., bell, tone, light, etc.) are presented that

*Address correspondence to this author at the Department of Psychology, College of Charleston, 57 Coming Street, Charleston, SC 29424, USA; Tel: (843) 953-8194; Fax: (843) 953-7151; E-mail: widholmj@cofc.edu predict and provide information about the occurrence of a US, they become Conditional Stimuli (CS; their ability to elicit a response is conditional on being predictive of a US) and the corresponding response is called the Conditional Response (CR). This process of conditioning CRs is referred to as acquisition. Modern Pavlovian theory views conditioning as an active process whereby the organism is an "information seeker" and conditioning results, not because stimuli are simply paired together, but rather because the CS allows the organism to better predict the US. In the absence of this relationship, conditioning is unlikely to obtain [15]. For example, when rats are exposed to shocks (US) that are always preceded by the signaling of a tone (CS), they exhibit a strong fear to the tone. However, rats exposed to many more shock trials that are not reliably correlated with a tone show very little fear to the tone, even though they are exposed to the same amount of tone-shock trials and receive more total shock trials [16]. In addition, research has shown that the associations that are formed can combine to produce more complex hierarchical associative structures indicating that Pavlovian conditioning produces more than just a static mental representation of the stimulus elements [15].

Following acquisition, the $\mathrm{CR}$ can be reduced or eliminated through the process of extinction in which the CS is repeatedly presented in the absence of US, resulting in the gradual loss of the CR. Fig. (1) diagrams a typical forward Pavlovian acquisition and extinction procedure and plots the theoretical (but typical) acquisition and extinction curves.

Our understanding of drug addiction and ability to predict the likelihood of relapse has been enhanced greatly by the application of Pavlovian models of learning [8, 9, 17, 18]. Within this paradigm, a drug of abuse (e.g., cocaine or heroin) is a US that innately elicits a response in the user and stimuli that are associated with drug use (drug syringe, needle, etc.) can become associated and elicit similar (or in some cases, opposite; see [19]) responses. For example, a drug-dependent patient that began injecting heroin (US) with 


\section{Acquisition}
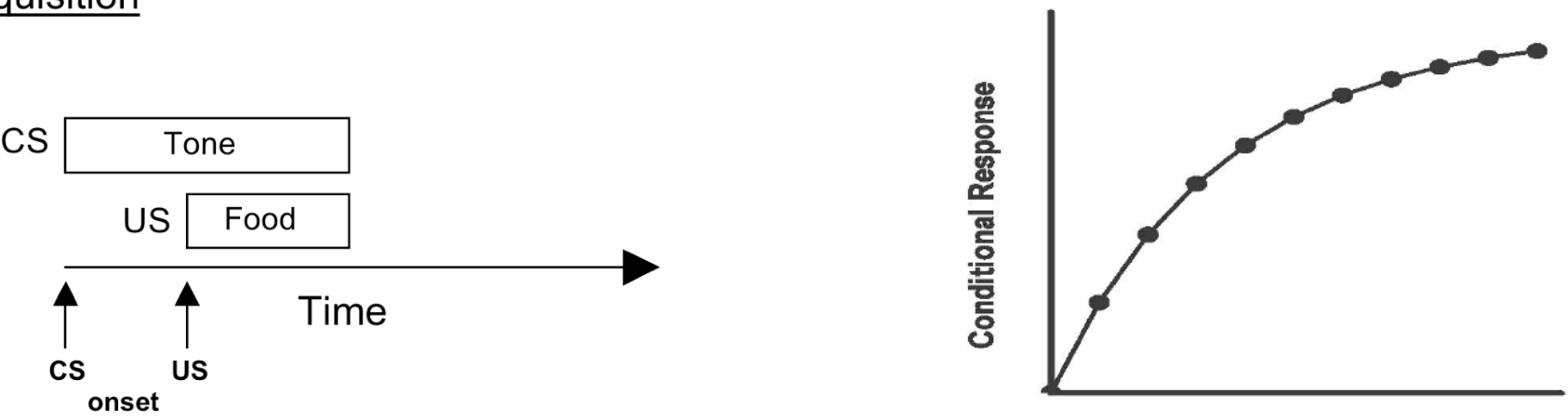

Trial

\section{Extinction}
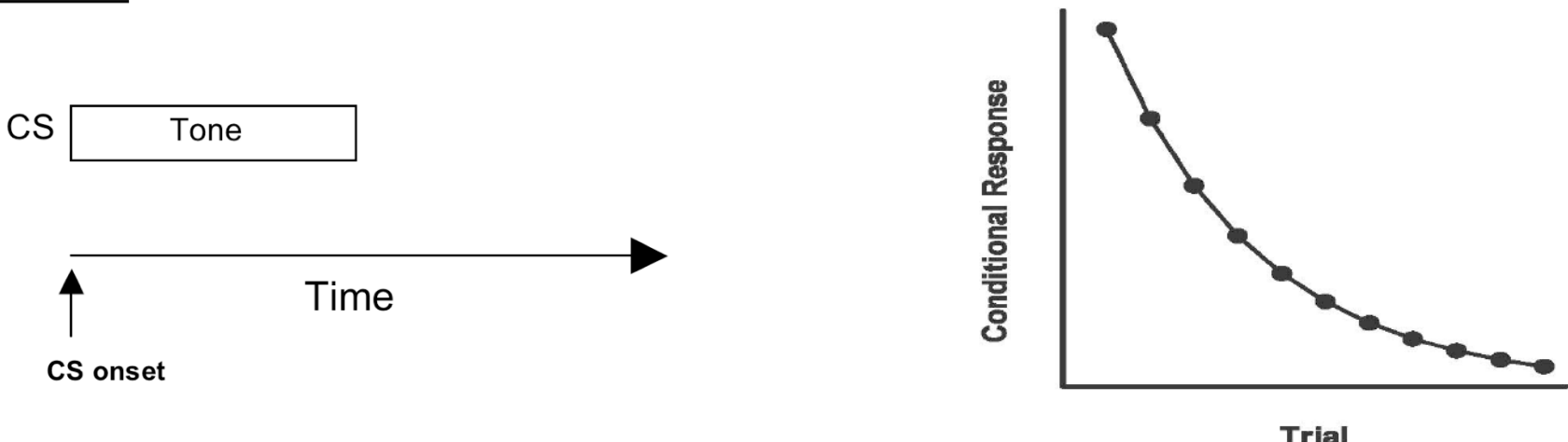

Fig. (1). Schematic depicting typical acquisition and extinction procedures with theoretical learning curves. Note: CS $=$ Conditional Stimulus; US = Unconditional Stimulus.

a friend (CS) may find that they have heroin cravings when they are in the presence of the friend or if they see others using similar drug-related paraphernalia (e.g., needle and syringe). Data from self-report and neurological studies consistently find increases on measures of craving in response to drug-related stimuli (e.g., [20, 21]). These cravings then serve to increase the likelihood (via negative reinforcement) of drug seeking behavior. Similar to findings from conditioning studies utilizing food [22] and/or shock [2], extinction procedures in which drug related stimuli are repeatedly presented but are not followed by the drug can be used to attenuate the CRs $[12,13,23]$. As such, cue-based drug treatment therapies $[11,24,25]$ based on extinction principles have been utilized to reduce cue-induced drug cravings and minimize the risks of relapse once the patient returns to the environment in which drug addiction occurred. However, these types of therapies are often of limited success, due in part to a misunderstanding of and/or the inability to control for relevant variables that affect extinction learning that may include any of the following; an underestimation of the role context plays in learning [2], insufficient time to allow for consolidation of extinction learning [26], and/or the inability to control for the pervasive threats to extinction that include renewal, spontaneous recovery, reinstatement, and/or rapid reacquisition [10].

Although it has been known since Pavlov's earliest experiments [1] that extinction doesn't completely erase the associations formed during acquisition because of the discovery that the $\mathrm{CR}$ recovers with time (spontaneous recovery), some early models of associative learning assumed that extinction involved the destruction of the original CS-US association (e.g., [27]). Although the assumption of associative erasure has since been dismissed by most, early cue-based therapies evolved from these assumptions and (perhaps due to this misattribution) have had limited success in preventing relapse in drug-dependent patients $[28,29]$. More recently, other threats to extinction have been uncovered; including renewal, reinstatement, and rapid reacquisition and each suggests that not only does (at least) some portion of the original association persist following extinction, they also suggest that new learning occurs during extinction that is context-dependent. What follow is a brief overview of each behavioral phenomenon and a discussion of its relevance for drug treatment.

\section{THREATS TO EXTINCTION}

\section{Renewal Effect}

The renewal effect refers to the reappearance (i.e., renewal) of the $\mathrm{CR}$ when the environment is changed from that used during extinction. Research on this phenomenon has uncovered compelling evidence on the importance of context in extinction learning (e.g., [7]). Stimuli that predict (and are temporally proximal to) the US become CSs while the background environmental cues present during learning that do not directly elicit the target CR become contextual cues $[2,4-7,22,30-32]$. For example, in an appetitive conditioning experiment [31] a tone (CS) was paired with food (US) in one animal test chamber (context A) to elicit a 
headjerking response (CR). Following acquisition, the rats underwent extinction in a different test chamber (context B) and were then tested in context $\mathrm{A}$ after the $\mathrm{CR}$ had been extinguished. Testing in context A caused a renewal of responding that was not evident in rats in which acquisition, extinction, and testing all occurred in the same context (A). This is an example of ABA renewal and it has been reliably demonstrated in appetitive (e.g., food; [31,33] and aversive conditioning (e.g., shock; $[2,30])$. Furthermore, AAB and $\mathrm{ABC}$ renewal have also been shown to occur $[2,22,33]$.

Bouton and colleagues have adopted a memory-based model of conditioning and attribute the renewal effect to a byproduct of acquisition-extinction ordering. Because extinction learning occurs after acquisition, the CS acquires multiple "meanings" (i.e., it both predicts the presence and absence of the US) which are stored as distinct memories [47]. The context is then used to disambiguate the CS to allow the recollection of the appropriate memory. In their view, the context doesn't directly control responding, but rather serves to set the occasion for which response is correct (see [34]; figure 9). That $\mathrm{AAB}$ and $\mathrm{ABC}$ renewal occurs argues against any interpretation of renewal due solely to excitatory conditioning of the context alone since neither context $\mathrm{B}$ (in $\mathrm{AAB}$ ) nor context $\mathrm{C}$ (in $\mathrm{ABC}$ ) was used for the conditioning trials. Furthermore, it highlights a consistent finding in extinction studies; the behavioral effects of extinction are context-specific whereas the effects of acquisition more readily generalize to other contexts.

The renewal effect has also been observed in animal models of drug self-administration. The possibility for ABA and $\mathrm{AAB}$ renewal was assessed in rats trained to selfadminister a heroin-cocaine mixture (i.e., a speedball) in the presence of discrete drug cues [35]. Following extinction to the drug cues in the same (A) or different (B) context, the rats were subsequently tested for renewal in either context. They found a powerful ABA renewal effect in their study, but (surprisingly) did not demonstrate $\mathrm{AAB}$ renewal [35]. Research with animals has reliably demonstrated ABA renewal with a variety of drugs including cocaine [13, 36], heroin [37], nicotine [38], and alcohol [39].

Because animal drug self-administration studies are an operant paradigm (i.e., the rats emit lever presses in order to receive drug infusions accompanied by discrete stimuli), one interpretation of the renewal effect in these studies is that the context is serving as an operant discriminative stimulus that signals the availability of reinforcement. From this perspective, responding renews not because of an uncovering of the original association (Pavlovian) but rather due to an increased tendency to respond while in the presence of stimuli that signal drug availability for responding. However, [36] manipulated their procedure to assess both Pavlovian and operant interpretations and concluded that the context served as a Pavlovian occasion setter and not as an operant discriminative stimulus. Furthermore, when alternative forms of reinforcement were available, the extent to which contextual renewal occurred was reduced [36].

The renewal effect has implications regarding drug treatment and relapse, suggesting that a drug addict who acquires a drug habit in one environment (e.g., home) and undergoes cue-exposure therapy in a clinic is likely to experience renewal of drug cravings when confronted with drug-associated stimuli when back home. The animal research indicates that when the environment for extinction and post-extinction testing are the same, renewal does not occur and this finding has been replicated in the human therapeutic literature. Cue-based exposure studies of spiderphobics [40-42], smokers [43], and social drinkers [44] have all found that that the CR is less likely to return if the test context and the extinction context match. Therefore, a similar reduction in the renewal of drug cravings might be obtained if therapy occurred in (or approximated) the environments in which drug use was learned. Unfortunately, few attempts appear to have been made to accomplish this with drug-dependent patients and the results appear equivocal [45].

In addition, it appears that renewal (and/or relapse) can be attenuated by conducting extinction sessions in multiple contexts. Rats that underwent extinction training in several novel contexts exhibited less of a renewal effect suggesting that the generalizability of extinction learning can be increased by conducting it in multiple contexts [46]. Lastly, evidence from [36] suggests that alternative forms of reinforcement for recovering addicts may help to minimize the extent to which renewal occurs.

\section{Spontaneous Recovery}

Following the extinguishing of the $\mathrm{CR}$, it can spontaneously reappear with the passage of time [1, 3, 47]. The importance of time as the critical variable is demonstrated in [3] in which rats were presented with two CSs (noise and light) that signaled the presentation of two possible USs (food or sucrose). Following acquisition, both stimuli were subjected to conditions of extinction and tested again one or eight days later. Spontaneous recovery occurred to the CS that was tested eight days following extinction but did not occur to the CS tested one day after extinction. Similarly, [48] observed similar temporal effects on spontaneous recovery in which rats tested six days after extinction demonstrated recovery of the CR while those that were tested 5 hours after extinction showed little recovery. Similar spontaneous recovery effects have also been demonstrated with drugs in animal models. Rats that underwent extinction exhibited higher levels of drug-related responding after 28 days of forced abstinence than after 7 days of forced abstinence [49].

Regarding the importance of temporal variables, the incubation effect refers to an increase in CR magnitude with increasing delays after acquisition. For example, [50] manipulated the delay between acquisition of cocaineassociated stimuli and the testing of those stimuli for 1 day, 1 month, 3 months, and 6 months and found that the tendency to respond in the presence of drug-associated CSs increased as a function of time since acquisition through the first 3 months [50]. This phenomenon has also been demonstrated in fear conditioning with enhancements in conditioned fear in rats that increase with the passage of time since acquisition [e.g., 51]. These data suggest that the passage of time, whether it occurs before extinction-based therapy (i.e., incubation), or after (spontaneous recovery) can be an important predictor of the tendency to relapse.

Additionally, the extent to which extinction trials are massed or spaced over time also appears to have an effect on 
the likelihood of spontaneous recovery. Rats trained with either massed (interval of 4 minutes between trials) or spaced ( 24 hours between trials) extinction trials and tested for fear 24 hours later differentially expressed spontaneous recovery with rats who underwent massed extinction procedures demonstrating recovery and rats who underwent spaced extinction procedures did not [52]. This effect appears to be due to (at least in part) to an asymmetry in temporal variables (i.e., temporal context) between extinction training and testing. For example, [53] manipulated the time between extinction trials in rats (four minutes $v s 16$ minutes between trials) and tested the rats for spontaneous recovery 16 minutes after extinction testing. Only the 4-minute ITI group showed recovery when tested 16 minutes after extinction suggesting that there was greater generalization between extinction and testing when the intervals in extinction and testing were similar.

The phenomenon of spontaneous recovery predicts that an abstinent drug-dependent patient is at risk of relapse that may increase with time [49]. It is unclear how long cravings can be elicited following extinction, but [21] observed cueinduced cravings for cocaine addicts following a 12 month period of abstinence. The behavioral research also suggests that spontaneous recovery could be minimized in abstinent patients by spacing the cue-exposure therapy trials over time both within and between each session to allow for spontaneous recovery to occur followed by further extinction training [10]. Meta-analytic studies have shown that the tendency to relapse in cocaine-addicted patients decreases with longer (i.e., $>=90$ days) as opposed to shorter (i.e., 21 days) treatment periods, further supporting the idea that extended treatment periods may be necessary to overcome the effects of cue-induced relapse [54].

Lastly, it has been shown in animal studies that presenting extinction-related cues during testing can attenuate spontaneous recovery, presumably by enhancing extinctionrelated memories [48, 55-58]. For example, [56] found that visual stimuli (house light off or illuminated keylight) presented prior to the CS (tone) during extinction could then be used as a cue to reduce the extent to which the spontaneous recovery of magazine entries (CR) occurred in rats when tested six days following the completion of extinction. These data suggest that providing abstinent patients cues related to extinction training might enhance extinctionrelated memories and serve as an effective tool in combating the tendency for spontaneous recovery [10].

\section{Reinstatement}

Reinstatement refers to the reappearance of the CR following post-extinction exposure to the US (or, in some cases, drug-related CSs). For example, following the extinction of fear in rats that had undergone tone (CS) shock (US) conditioning, independent presentations of the US can reinstate fear in the rats without any need for further CS-US pairings [59]. It was concluded that the data support the idea that individual events (i.e., memories) within the learned association are susceptible to degradation during extinction and that independent presentations of the US reenergize the memory and cause reinstatement. Moreover, presentations of other USs that were not used in conditioning (but were similar to the US used in conditioning) were found to result in reinstatement of the CR [59]. For example, when a loud, unpredictable noise was substituted for shock as the reinstating stimulus, the $\mathrm{CR}$ was reinstated to the CS.

More recent investigations have revealed that reinstatement may be another form of the renewal effect that is contextually-dependent [60]. Rats that underwent fear conditioning and extinction in a conditioning chamber (context A) did not show the reinstatement effect when the US was presented in the conditioning chamber (context A) but tested in lever-press operant chambers (context B). Conversely, rats that were subjected to the US in the lever press operant chambers showed a large reinstatement effect when tested in this context. The idea that extinction and reinstatement effect changes to individual elements within the original association [59] are not supported by these findings [60] but rather suggest that the reinstatement effect is a function of contextual conditioning brought on by the pairing of the reinstating US and the context in which it occurs. That is, rather than interpreting the reinstatement effect as a re-energizing of elements within the original CSUS association, an association between the context and US is formed. Such an association is absent during extinction which serves to change the extinction context. Thus, the presence of the reinstating US makes the context more like it was during conditioning making reinstatement analogous to an ABA renewal effect [see 61].

Animal research using drugs as reinforcers have found effects identical to those obtained in the learning literature. Using the conditioned place preference (CPP) paradigm in rats, following the extinction of a preference for a particular location that was associated with cocaine injections, the preference could be reinstated following an injection of cocaine without any further conditioning trials [62]. Animal drug self-administration paradigms have found similar reinstatement effects. Early demonstrations of drug reinstatement in monkeys [63] and rats $[64,65]$ found that priming injections following extinction elevated responding to levels seen during acquisition. These results corroborate the data from abstinent drug-dependent patients who report experiencing stronger drug cravings shortly after acute exposure to a drug than before [66]. Additionally, [64] demonstrated a similar generalization effect to that obtained by [59] and were able to induce reinstatement with drugs of similar interoceptive effects of cocaine (e.g., amphetamine, morphine). However, the conclusions of [2,4-7] suggest that the environment in which the priming exposure is given is an important predictor of the likelihood of drug-induced relapse and the data from some animal drug self-administration studies support this proposition (e.g., [67]).

\section{Rapid Reacquisition}

Rapid reacquisition, as the name implies, refers to the enhanced rate of $\mathrm{CR}$ elicitation to a previously extinguished CS. While it seems intuitive to assume that additional CS-US pairings following extinction would result in more rapid reacquisition (and early research supported this idea; [68, 69]), the early data now appear ambiguous as alternative interpretations to rapid reacquisition have been proposed (e.g., spontaneous recovery, renewal). Furthermore, recent research has discovered that some conditioning preparations result in rapid reacquisition and others do not [70]). For 
example, rapid reacquisition has been demonstrated in conditioning reflexive movements of the nictating membrane in rabbits [71] and in a contextual fear conditioning procedure [72]. However, [70] produced both slow and rapid reacquisition in an appetitive conditioning procedure and attributed the difference (in part) to the number of trials needed during acquisition with large numbers of trials creating rapid reacquisition and fewer acquisition trials, rapid reacquisition was unlikely. Additionally, reacquisition can be slowed following large numbers of extinction trials [72]. It has been proposed that rapid reacquisition is likely another form of ABA renewal in which the contexts differ in terms of US presentation [7]. For example, rats learn that CS-US pairings are part of acquisition and CS-only trials are part of extinction and this difference in which the trials unfold serve as a contextual background. If correct, presentation of CS-US trials during reacquisition would "transport" the rat to the acquisition context and responding would be renewed (i.e., ABA renewal). Consistent with this prediction, when some CS-US trials were added to extinction conditions to equate this aspect of context, reacquisition was slowed [73].

While the research is still unclear about the extent to which rapid reacquisition reflects a true enhancement of learning that is not attributable to other extinction related phenomenon (e.g., spontaneous recovery), some of the research suggests that this phenomenon may be applicable to the treatment of drug-dependent patients. As [70] have suggested, the extent to which rapid reacquisition occurs appears dependent on the number of trials used during acquisition. Many drug addicts come to treatment with many years of drug experience and many experiences over which stimuli are conditioned (e.g., over learning). Based on the findings of [72] and others, massive numbers of extinction trials may be necessary in this situation to reduce the possibility of a rapid relearning of drug-related associations following treatment.

\section{SUMMARY}

The renewed interest in animal extinction learning has provided novel ways to interpret the variables that affect extinction-based therapies for treatment of drug-dependence. The "threats to extinction" indicate that much of what was learned during acquisition remains after extinction and these threats model common forms of relapse in drug addicts. Neurobiological, neuropharmacological, and/or neurophysiological research designed to understand and/or facilitate the extinction process has clinical implications and the behavioral research suggests that treatments capable of enhancing extinction-related memories (e.g., [2,4-7]) or generalization of learning beyond the extinction context (e.g., [46]) may enhance the effectiveness of those treatments.

\section{REFERENCES}

[1] Pavlov IP. Conditioned reflexes. Oxford: Oxford University Press 1927.

[2] Bouton ME, Bolles RC. Contextual control of the extinction of conditioned fear. Learn Motiv 1979; 10: 445-66.

[3] Rescorla RA. Spontaneous recovery after Pavlovian conditioning with multiple outcomes. Anim Learn Behav 1997; 25: 99-107.

Bouton ME. Context, time, and memory retrieval in the interference paradigms of Pavlovian learning. Psychol Bull 1993; 114: 80-99.

[5] Bouton ME. Context, ambiguity, and classical conditioning. Curr Direct Psychol Sci 1994; 3: 49-53.

[6] Bouton ME. Conditioning, remembering, and forgetting. J Exp Psychol Anim B 1994; 20: 219-31.

[7] Bouton ME. Context and behavioral processes in extinction. Learn Mem 2004; 11: 485-94.

[8] Siegel S. Classical conditioning, drug tolerance, and drug dependence. In: Smart RG, Glaser FB, Israel Y, Kalant H, Popham RE, Schmidt W, Eds. Research advances in alcohol and drug problems. New York, Plenum 1983; pp. 207-46.

[9] Siegel S. Pavlovian conditioning and heroin overdose: Reports by overdose victims. Br Psychonom Soc 1984; 22: 428-30.

[10] Conklin CA, Tiffany ST. Applying extinction research and theory to cue-exposure addiction treatments. Addiction 2002; 97: 155-67.

[11] Heather N, Bradley BP. Cue exposure as a practical treatment for addictive disorders: Why are we waiting? Addict Behav 1990; 15: $335-7$.

[12] Gass JT, Osborne MPH, Watson NL, Brown JL, Olive MF mGluR5 antagonism attenuates methamphetamine reinforcement and prevents reinstatement of methamphetamine-seeking behavior in rats. Neuropsychopharmacology 2008; 34: 820-33.

[13] Fuchs RA, Evans KA, Ledford CC, Parker MP, Case JM, Mehta RH, See RE. The role of the dorsomedial prefrontal cortex, basolateral amygdala, and dorsal hippocampus in contextual reinstatement of cocaine seeking in rats. Neuropsychopharmacology 2005; 30: 296-309.

[14] Davis M, Ressler K, Rothbaum BO, Richardson R. Effects of Dcycloserine on extinction: Translation from preclinical to clinical work. Biol Psychiatry 2006; 60: 369-75.

[15] Rescorla RA. Pavlovian conditioning: It's not what you think it is. Am Psychol 1988; 43: 151-60.

[16] Rescorla RA. Probability of shock in the presence and absence of CS in fear conditioning. J Comp Physiol Psychol 1968; 66: 1-5.

[17] Wikler A. Dynamics of drug dependence, implications of a conditioning theory for research and treatment. Arch Gen Psychiatry 1973; 28: 611-6.

[18] Siegel, S. Evidence from rats that morphine tolerance is a learned response. J Comp Physiol Psychol 1975; 89: 498-506.

[19] Siegel S, Hinson RE, Krank MD, McCully J. Heroin "overdose" death: contribution of drug-associated environmental cues. Science 1982; 216: 436-7.

[20] Childress AR, Mozley PD, McElgin W, Fitzgerald J, Reivich M, O’Brien CP. Limbic activation during cue-induced cocaine craving. Am J Psychiatry 1999; 156: 11-8.

[21] Franken IH, de Haan HA, van der Meer CW, Haffmans PM, Hendriks VM. Cue reactivity and effects of cue exposure in abstinent posttreatment drug users. J Subst Abuse Treat 1999; 16: 81-5.

[22] Bouton ME, Ricker ST. Renewal of extinguished responding in a second context. Anim Learn Behav 1994; 22: 317-24.

[23] Siegel S, Sherman JE, Mitchell D. Extinction of morphine analgesic tolerance. Learn Motiv 1980; 11: 289-301.

[24] Ehrman RN, Robbins SJ, Childress AR, Goehl L, Hole AV, O'Brien CP. Laboratory exposure to cocaine cues does not increase cocaine use by outpatient subjects. J Subst Abuse Treat 1998; 15: 431-5.

[25] O'Brien CP, Childress AR, McLellan T, Ehrman R. Integrating systematic cue exposure with standard treatment in recovering drug dependent patients. Addict Behav 1990; 15: 355-65.

[26] Quirk GJ, Mueller D. Neural mechanisms of extinction learning and retrieval. Neuropsychopharmacology 2008; 33: 56-72.

[27] Rescorla RA, Wagner AR. A theory of Pavlovian conditioning: Variations in the effectiveness of reinforcement and nonreinforcement. In: Black AH, Prokasy WK, Eds. Classical conditioning II: current research and theory. New York, Appleton-Century-Crofts 1972; pp. 64-99.

[28] Dawe S, Powell JH, Richards D, et al. Does post-withdrawal cue exposure improve outcome in opiate addiction? A controlled trial. Addiction 1993; 88: 1233-45.

[29] Powell J, Gray J, Bradley BP. Subjective craving for opiates: Evaluation of a cue exposure protocol for use with detoxified opiate addicts. Br J Clin Psychol 1993; 32: 39-53. 
[30] Bouton ME, King DA. Contextual control of the extinction of conditioned fear: tests for the associative value of the context. J Exp Psychol Anim B 1983; 9: 248-65.

[31] Bouton ME, Peck CA. Context effects on conditioning, extinction, and reinstatement in an appetitive conditioning preparation. Anim Learn Behav 1989; 17: 188-98.

[32] Bouton ME, Nelson JB, Rosas JM. Stimulus generalization, context change, and forgetting. Psychol Bull 1999; 125: 171-86.

[33] Rescorla RA. Within-subject renewal in sign-tracking. Q J Exp Psychol 2008; 61: 1793-802.

[34] Bouton ME, Nelson JB. Context-specificity of target vs feature inhibition in a feature negative discrimination. J Exp Psychol Anim B 1994; 20: 51-65.

[35] Crombag HS, Shaham Y. Renewal of drug seeking by contextual cues after prolonged extinction in rats. Behav Neurosci 2002; 116 : 169-73.

[36] Kearns DN, Weiss SJ. Contextual renewal of cocaine seeking in rats and its attenuation by the conditioned effects of an alternative reinforcer. Drug Alcohol Depend 2007; 90: 193-202.

[37] Bossert JM, Liu SY, Lu L, Shaham Y. A role of ventral tegmental area glutamate in contextual cue-induced relapse in heroin seeking. J Neurosci 2004; 24: 10726-30.

[38] Diergaarde L, de Vries W, Raasø H, Schoffelmeer ANM, De Vries TJ. Contextual renewal of nicotine seeking in rats and its suppression by the cannabinoid-1 receptor antagonist Rimonabant (SR141716A). Neuropharmacology 2008; 55: 712-6.

[39] Chaudhri N, Sahuque LL, Janak PH. Context-induced relapse of conditioned behavioral responding to ethanol cues in rats. Biol Psychiatry 2008; 64: 203-10.

[40] Mystkowski JL, Craske MG, Echiverri AM. Treatment context and return of fear in spider phobia. Behav Ther 2002; 33: 399-416.

[41] Mystkowski JL, Mineka S, Vernon LL, Zinbarg RE. Changes in caffeine states enhance return of fear in spider phobia. J Consult Clin Psychol 2003; 71: 243-50.

[42] Mineka S, Mystkowski JL, Hladek D, Rodriguez BI. The effects of changing contexts on return of fear following exposure therapy for spider fear. J Consult Clin Psychol 1999; 67: 599-604.

[43] Thewissen R, Snijders SJBD, Havermans RC, van den Hout M, Jansen A. Renewal of cue-elicited urge to smoke: implications for cue exposure treatment. Behav Res Ther 2006; 44: 1441-9.

[44] Collins BN, Brandon TH. Effects of extinction context and retrieval cues on alcohol cue reactivity among nonalcoholic drinkers. J Consult Clin Psychol 2002; 70: 390-7.

[45] Kasvikis Y, Bradley B, Powell J, Marks I, Gray JA. Postwithdrawal exposure treatment to prevent relapse in opiate addicts: a pilot study. Int J Addict 1991; 26: 1187-95.

[46] Chelonis JJ, Calton JL, Hart JA, Schachtman TR. Attenuation of the renewal effect by extinction in multiple contexts. Learn Motiv 1999; 30: 1-14.

[47] Robbins SJ. Mechanisms underlying spontaneous recovery in autoshaping. J Exp Psychol Anim Behav Process 1990; 16: 235-49.

[48] Brooks DC, Bouton ME. A retrieval cue for extinction attenuates spontaneous recovery. J Exp Psychol Anim Behav Process 1993; 19: 77-89.

[49] DiCiano P, Everitt BJ. Reinstatement and spontaneous recovery of cocaine-seeking following extinction and different durations of withdrawal. Behav Pharmacol 2002; 13: 397-405.

[50] Lu L, Grimm JW, Dempsey J, Shaham Y. Cocaine seeking over extended withdrawal periods in rats: different time courses of responding induced by cocaine cues vs cocaine priming over the first 6 months. Psychopharmacology (Berl) 2004; 176: 101-8.

[51] Pickens CL, Golden SA, Adams-Deutsch T, Nair SG, Shaham Y. Long-lasting incubation of conditioned fear in rats. Biol Psychiatry 2009; 65: 881-6.
[52] Li SH, Westbrook RF. Massed extinction trials produce better short-term but worse long-term loss of context conditioned fear responses than spaced trials. J Exp Psychol Anim Behav Process 2008; 34: 336-51.

[53] Bouton ME, Gutierrez AG. Intertrial interval as a contextual stimulus. Behav Process 2006; 71: 307-17.

[54] Simpson DD, Joe GW, Fletcher BW, Hubbard RL, Anglin MD. A national evaluation of treatment outcomes for cocaine dependence. Arch Gen Psychiatry 1999; 56: 507-14.

[55] Brooks DC, Bouton ME. A retrieval cue for extinction attenuates response recovery (renewal) caused by a return to the conditioning context. J Exp Psychol Anim Behav Process 1994; 20: 366-79.

[56] Brooks DC. Recent and remote extinction cues reduce spontaneous recovery. Q J Exp Psychol 2000; 53B: 25-58.

[57] Brooks DC, Vaughan JM, Freeman AJ, Woods AM. An extinction cue reduces spontaneous recovery of ataxic ethanol tolerance in rats. Psychopharmacology (Berl) 2004; 176: 256-65.

[58] Brooks DC. Alcohol ataxia tolerance: extinction cues, spontaneous recovery, and relapse. Int J Comp Psychol 2005; 18: 141-53.

[59] Rescorla RA, Heth CD. Reinstatement of fear to an extinguished conditioned stimulus. J Exp Psychol Anim Behav Process 1975; 104: 88-96.

[60] Bouton ME, Bolles RC. Role of conditioned contextual stimuli in reinstatement of extinguished fear. J Exp Psychol Anim Behav Process 1979; 5: 368-78.

[61] Bouton ME, Westbrook RF, Corcoran KA, Maren S. Contextual and temporal modulation of extinction: behavioral and biological mechanisms. Biol Psychiatry 2006; 60: 352-60.

[62] Mueller D, Stewart J. Cocaine-induced conditioned place preference: reinstatement by priming injections of cocaine after extinction. Behav Brain Res 2000; 115: 39-47.

[63] Stretch R, Gerber GJ. Drug-induced reinstatement of amphetamine self-administration behavior in monkeys. Can J Psychol 1973; 27 : 168-77.

[64] de Wit H, Stewart J. Reinstatement of cocaine-reinforced responding in the rat. Psychopharmacology (Berl) 1981; 75: 13443.

[65] de Wit H, Stewart J. Reinstatement of heroin-reinforced responding in the rat. Psychopharmacology (Berl) 1983; 79: 29-31.

[66] Meyer R, Mirin S. The heroin stimulus: implications for a theory of addiction. New York: Plenum Press 1979.

[67] Wing VC, Shoaib M. Contextual stimuli modulate extinction and reinstatement in rodents self-administering intravenous nicotine. Psychopharmacology (Berl) 2008; 200: 357-65.

[68] Hoehler FK, Kirschenbaum DS, Leonard DW. The effects of overtraining and successive extinctions upon nictitating membrane conditioning in the rabbit. Learn Motiv 1973; 4: 91-101.

[69] Frey PW, Butler CS. Extinction after aversive conditioning: an associative or nonassociative process? Learn Motiv 1977; 8: 1-17.

[70] Ricker ST, Bouton ME. Reacquisition following extinction in appetitive conditioning. Anim Learn Behav 1996; 24: 423-36.

[71] Napier RM, Macrae M, Kehoe EJ. Rapid reacquisition in conditioning of the rabbit's nictating membrane response. J Exp Psychol Anim Behav Process 1992; 18: 182-92.

[72] Leung HT, Bailey GK, Laurent V, Westbrook RF. Rapid reacquisition of fear to a completely extinguished context is replaced by transient impairment with additional extinction training. J Exp Psychol Anim Behav Process 2007; 33: 299-313.

[73] Bouton ME, Woods AM, Pineño O. Occasional reinforced trials during extinction can slow the rate of rapid reacquisition. Learn Motiv 2004; 35: 371-90. 